\section{Multi-locus sequence typing and virulence profile in Bacillus cereus sensu lato strains isolated from dairy products}

\author{
Angelica Bianco, Loredana Capozzi, \\ Angela Miccolupo, Simona Iannetti, \\ Maria Luisa Danzetta, \\ Laura Del Sambro, Marta Caruso, \\ Gianfranco Santagada, Antonio Parisi
}

Experimental Zooprophylactic Institute of Apulia and Basilicata, Foggia, Italy

\begin{abstract}
Members of Bacillus cereus group are important food contaminants and they are of relevant interest in food safety and public heath due to their ability to cause two distinct forms of food poisoning, emetic and diarrhoeal syndrome. In the present study, 90 strains of $B$. cereus isolated from dairy products, have been typed using Multilocus Sequence Typing (MLST) analysis and investigated for the occurrence of 10 enterotoxigenic genes ( $h b l A, h b l C, h b l D$, nheA, nheB, nhe $C$, cytK, entFM, entS and $b c e T)$ and one emetogenic gene (ces), to determine their genetic diversity. A total of 58 sequence types were identified and among these 17 were signalled as new profiles. Among the virulence genes, the majority of our strains carried the entS $(92 \%)$, entFM (86\%), nhe $(82 \%)$ and cytK $(72 \%)$ genes. All remaining genes were identified in at least one strain with different prevalence, stressing the genetic diversity, how even the different grade of pathogenicity of $B$. cereus isolated from dairy products.
\end{abstract}

\section{Introduction}

Bacillus cereus (B. cereus) is Grampositive, rod shaped, endospore-forming, facultative anaerobic organism of genus Bacillus. Several closely related Bacillus species share a significant genetic similarity with $B$. cereus, thus they are included in a heterogeneous $B$. cereus group (Chang et al., 2003). B. cereus group is ubiquitous in the environment, particularly soil and water, because of the resistance of its spores to adverse environmental conditions (Brillard et al., 2015). It has been isolated from a variety of foods, including meat, rice, pasta, condiments, raw fruits, spices and vegetables (Harmon and Kautter, 1991), chinese "take-out foods" (Beuchat Ma-Lin et al.,
1980), cooked chicken (López et al., 2015), milk and dairy products (Christiansson, Bertilsson, and Svensson, 1999).

Some members of $B$. cereus group (i.e. $B$. cereus and $B$. thuringiensis) can cause human gastrointestinal diseases, the emetic and diarrhoeal syndromes. The emetic syndrome is characterised by vomiting, followed by diarrhoea (Beecher and Wong, 1994). This syndrome is caused by the ingestion of the cereulide toxin, preformed in the food (Agata et al., 2002). The diarrhoeal syndrome, is characterized by watery diarrhoea associated with abdominal pain (Granum and Lund, 1997) and it is caused by the ingestion of vegetative cells of $B$. cereus present in food, that colonize the small intestine before starting the enterotoxins production (i.e. non-haemolytic enterotoxin NHE, haemolysin BL, cytotoxin K) (Granum and Lund, 1997). Additionally, enterotoxin FM (EntFM), enterotoxin T (BceT) and enterotoxin S (EntS) have been also described as potential diarrheal toxins, although it is not clear the role they play in the pathogenesis of the disease (Hendriksen et al., 2006).

The aim of this study was to identify the virulence genes and to determine the sequence type of $B$. cereus sensu lato (s.l.) strains isolated from dairy products using Multi-locus Sequence Typing (MLST).

\section{Materials and Methods}

\section{Multi-locus sequence typing}

A total of 90 strains of $B$. cereus s.l. were isolated from dairy products: $64 \%$ (57/90) from caciocavallo, 25\% (22/90) from mozzarella, 2\% (2/90) from sheep's cheese; $2 \%(2 / 90)$ from butter; $2 \%(2 / 90)$ from milk; $1 \%(1 / 90)$ from Buffalo mozzarella; $1 \%$ (1/90) from cacioricotta; $1 \%$ (1/90) from cream cheese; $1 \%$ (1/90) from caciotta; $1 \%(1 / 90)$ from yogurt. All the samples were collected from 2016 to 2018.

Genomic DNA was purified using DNeasy Blood and Tissue Kit (Qiagen, Hilden, Germany), according to manufacturer's protocol. The MLST analysis was performed as described elsewhere (http://www.pubmlst.org/bcereus/).

DNA sequencing was performed on both strands using ABI PRISM Big Dye ${ }^{\mathrm{TM}}$ Terminator Cycle 3.1 sequencing kit cycle (Life Technologies) according to the manufacture's instruction and analyzed by ABI Prism 3130 Genetic Analyzer (Applied BiosystemThermoScientific inc.). The sequence of each allele was imported and assembled by Bionumerics v7.5 software (Applied Maths, Belgium), and then com-
Correspondence: Antonio Parisi, Experimental Zooprophylactic Institute of Apulia and Basilicata, Foggia, Italy. Tel.: +39.080.4057858; Fax: +39.080.4057753. E-mail: antonio.parisi@izspb.it

Key words: Bacillus cereus; food poisoning; Multi-locus Sequence Typing; toxin genes.

Contributions: All the authors contributed equally.

Conflict of interest: The authors declare no conflict of interest.

Funding: Fondi Ricerca Corrente Ministero della Salute IZS PB 04/16 RC

Received for publication: 15 July 2020

Revision received: 9 August 2020

Accepted for publication: 28 August 2020.

This work is licensed under a Creative Commons Attribution-NonCommercial 4.0 International License (CC BY-NC 4.0).

(C) Copyright: the Author(s), 2020

Licensee PAGEPress, Italy

Italian Journal of Food Safety 2020; 9:8401

doi:10.4081/ijfs.2020.8401

pared to those available in the MLST database. New allele sequences were submitted to the $B$. cereus MLST database.

\section{Virulence genes detection}

The presence of potential virulence genes $h b l A, h b l C$, hblD, nhe A, nheB, nheC, $c y t K$, entFM, ent $S$, bceT and ces was evaluated by PCR using primers listed in Table 1 (Asano et al., 1997; Guinebretière et al., 2002; Fagerlund et al., 2004) and followed the procedure described in Ghelardi et al., 2002 and Fricker et al., 2007

\section{Results}

In our study we collected a total of $90 \mathrm{~B}$. cereus s.l. strains isolated from different dairy products (Table 2). The MLST profiles of all isolates are shown in Table 2. A total of 58 STs were identified with ST 1986 as the most frequent. Additionally, 17 STs never described before were identified (Table 2). Thirteen STs belonged to 7 clonal complexes (CC). The major clonal complex was represented by the CC-142, that includes two STs, ST4 and ST2010 (Table 2). The CC-23 included three STs, ST197, ST2013 and ST2033; each CC-111, CC-142 and CC-365 included two STs: ST562 and ST800, ST4 and ST2010, ST1263 and ST2084, respectively. The CC-97 and CC- 
205 included each only one ST, 1578 and 255 , respectively. The ST1986 was the most frequent ST ad it was detected only in caciocavallo. When we considered the distribution of STs among the samples collected in this study (including samples with two or more strains isolated), we identified 34 different STs in caciocavallo, $15 \mathrm{STs}$ in mozzarella, 2 STs were identified in both butter and milk and sheep's cheese. Only two STs, ST92 and ST142, were identified in more than one dairy product.

All strains analyzed in this study were tested for the presence or absence of eleven virulence genes; the results were summarized on Table 2. Three genes encoding enterotoxin $\mathrm{HBL}$ were identified in $58 \%$ (53/90) of strains, with different prevalence: the $h b l A, h b l C$ and $h b l D$ were identified in $67 \%(60 / 90), 64 \%(58 / 90)$ and $79 \%(71 / 90)$ of the strains, respectively. Similarly, the cluster of $n$ he genes was identified in $84 \%$ (76/90) of the strains with different prevalence between genes: the nheA, nheB and nhe $C$ genes were identified in $92 \%(83 / 90)$, $93 \%(84 / 90)$ and $90 \%(81 / 90)$ of the strains, respectively. The cytK, entFM, bceT and ent $S$ genes were identified in $72 \%(65 / 90)$, $86 \%(77 / 90), 64 \%(59 / 90)$ and $92 \%(83 / 90)$ of the strains, respectively. Only the $10 \%$ (9/90) of the strains carried the ces gene.

\section{Discussion and Conclusions}

B. cereus s.l. is a ubiquitous bacterium in the environment that can be present in a wide range of foodstuffs. It is prevalent in soil from cattle grazing fields, raw milk and dairy products. In this study we describe the characterization and the pathogenic potential of a collection of $90 \mathrm{~B}$. cereus strains isolated from dairy products. Based on MLST profiles our strains showed a high genetic diversity. Indeed, on a total of 90 isolates 58 MLST profiles were identified, of which 17 were new ST profiles and were submitted in available online database. The ST1986 was the most frequent ST detected and it was found only in the strains isolated from caciocavallo. Interestingly, only the ST92 and ST142 were identified in more than one dairy product; all the other 56STs were detected in a particular dairy product variety. Among previously described STs, ten (ST4, ST12, ST15, ST24, ST26, ST33, ST164, ST205, ST1063 and ST1097) were identified in milk or milk products (Cardazzo et al., 2008; Didelot, Barker, Falush, and Priest, 2009; Yang et al., 2017). The remaining $31 \mathrm{STs}$ were isolated in other foods or in the environment (Didelot et al., 2009; Priest et al., 2004).

Different virulence genes have been associated to the pathogenic power of $B$. cereus, the Enterotoxins BL (HBL), NonHemolytic Enterotoxin (NHE), cytotoxin K, and enterotoxin FM are primarily associated to diarrheal disease (Madeira et al., 2015 Sergeev et al., 2006); evidence suggests that the enterotoxin EntS and BceT also contribute to the severity of diarrheal illness Agata et al., 1995; Castiaux et al., 2016; whereas the ability of $B$. cereus s.l. to synthesize the heat stable cereulide protein is connected to the emetic disease (EhlingSchulz et al., 2005). In our study we found that the non-emetic strains $(90 \%)$ were more numerous than the emetic strains $(10 \%)$. This finding agreed with previous studies, which showed a low prevalence rate of emetic toxin gene ces in B. cereus s.l. isolated from milk, dairy product and other various food sources (Yim et al., 2015; Owusu-Kwarteng et al., 2017). All these data seem to suggest that emetic toxin gene is rare among $B$. cereus s.l. strains, but an infection supported by this specific toxin is considered to be the worst-case scenario (Wetterhall et al., 2019). Generally, B. cereus s.l. strains related with emetic food poisoning were associated with ST26 (Ehling-Schulz et al., 2005). Based on the genetic profile of our strains, we not only corroborated this thesis, but we identified additionally five STs (ST4, ST33, ST1986, ST2045 and ST2062) as potential emetic strains. Interestingly, among these strains, those belonging to ST26 did not carried HBL gene complexes, as previous reported (Ehling-Schulz et al., 2005); among these, in the BC003 strain we identified only two virulence genes, ces and entS. Similarly, to ST26, both the strains belonged to ST2045 did not carried HBL gene complexes. Among the non-emetic B. cereus s.l. strains, we found a high prevalence rate $(\geq 60 \%)$ of all enterotoxin genes investigated. The higher prevalence rate was related to entS gene, identified in $93 \%$ of all strains, followed by entFM gene (83\%), nhe gene complex $(82 \%)$, cytK gene $(72 \%)$, bceT gene $(66 \%)$ and hblACD gene complex

Table 1. Sequence of PCR primers targeting eleven virulence genes used in this study.

\begin{tabular}{|c|c|c|c|}
\hline Target & Primer name & Primer sequence $\left(5^{\prime}-3^{\prime}\right)$ & Reference \\
\hline hblA & $\begin{array}{l}\text { hblA_F } \\
\text { HBLA_R }\end{array}$ & $\begin{array}{l}\text { GTGCAGATGTTGATGCCGAT } \\
\text { ATGCCACTGCGTGGACATAT }\end{array}$ & (Guinebretière et al., 2002) \\
\hline$h b l C$ & $\begin{array}{l}\text { L2A_F } \\
\text { L2B_R }\end{array}$ & $\begin{array}{l}\text { AATGGTCATCGGAACTCTAT } \\
\text { CTCGCTGTTCTGCTGTTAAT }\end{array}$ & (Guinebretière et al., 2002) \\
\hline$h b l D$ & $\begin{array}{l}\text { L1A_F } \\
\text { L1B_R }\end{array}$ & $\begin{array}{l}\text { AATCAAGAGCTGTCACGAAT } \\
\text { CACCAATTGACCATGCTAAT }\end{array}$ & (Guinebretière et al., 2002) \\
\hline nheA & $\begin{array}{l}\text { nheA_F } \\
\text { nheA_R }\end{array}$ & $\begin{array}{c}\text { TACGCTAAGGAGGGGCA } \\
\text { GTTTTTATTGCTTCATCGGCT }\end{array}$ & (Guinebretière et al., 2002) \\
\hline nheB & $\begin{array}{l}\text { nheB_F } \\
\text { nheB_R }\end{array}$ & $\begin{array}{l}\text { CTATCAGCACTTATGGCAG } \\
\text { ACTCCTAGCGGTGTTCC }\end{array}$ & (Fagerlund et al., 2004) \\
\hline nheC & $\begin{array}{l}\text { nheC_F } \\
\text { nheC_R }\end{array}$ & $\begin{array}{c}\text { CGGTAGTGATTGCTGGG } \\
\text { CAGCATTCGTACTTGCCAA }\end{array}$ & (Fagerlund et al., 2004) \\
\hline cytK & $\begin{array}{l}\text { cytK_F } \\
\text { cytK_R }\end{array}$ & $\begin{array}{l}\text { ACAGATATCGGKCAAAATGC } \\
\text { TCCAACCCAGTTWSCAGTTC }\end{array}$ & (Ghelardi et al., 2002) \\
\hline entFM & $\begin{array}{l}\text { entFM_F } \\
\text { entFM_R }\end{array}$ & $\begin{array}{l}\text { ATGAAAAAAGTAATTTGCAGG } \\
\text { TTAGTATGCTTTTGTGTAACC }\end{array}$ & (Asano et al., 1997) \\
\hline$b c t E$ & $\begin{array}{l}\text { entT_F } \\
\text { entT_R }\end{array}$ & $\begin{array}{l}\text { GCTACGCAAAAACCGAGTGGTG } \\
\text { AATGCTCCGGACTATGCTGACG }\end{array}$ & (Agata et al., 1995) \\
\hline Ces & $\begin{array}{l}\text { ces_F } \\
\text { ces_R }\end{array}$ & $\begin{array}{l}\text { GGTGACACATTATCATATAAGGTG } \\
\text { GTAAGCGAACCTGTCTGTAACAACA }\end{array}$ & (Fricker et al., 2007) \\
\hline
\end{tabular}


Table 2. Source, clonal complex (CC), sequence type (ST) and enterotoxin and emetic toxin genes identified in B. cereus s.l. isolated from dairy products. The new STs identified for the first time were indicated with asterisks.

\begin{tabular}{|c|c|c|c|c|c|c|c|c|c|c|c|c|c|c|}
\hline ID sample & Source & $\mathrm{CC}$ & ST & hblA & hblc & hblD & nheA & $\begin{array}{l}\text { Virule } \\
\text { nheB }\end{array}$ & $\begin{array}{l}\text { nce ge } \\
\text { nheC }\end{array}$ & $\begin{array}{l}\text { es } \\
\text { cytk }\end{array}$ & entFM & bceT & entS & ces \\
\hline $\mathrm{BC} 001$ & Caciocavallo & & $1967^{*}$ & + & + & + & + & + & + & + & + & + & + & - \\
\hline BC002 & Mozzarella & & 26 & - & - & - & + & + & + & - & + & - & + & + \\
\hline $\mathrm{BC} 003$ & Mozzarella & & 26 & - & - & - & - & - & - & - & - & - & + & + \\
\hline BC004 & Caciocavallo & & 26 & - & - & - & + & + & + & - & + & - & + & + \\
\hline BC005 & Caciocavallo & & 34 & - & - & - & + & + & - & + & + & + & + & - \\
\hline $\mathrm{BC} 006$ & Caciocavallo & & 33 & + & + & + & + & + & + & + & + & + & + & + \\
\hline $\mathrm{BC} 007$ & Caciocavallo & & 509 & - & - & + & + & + & + & + & - & + & + & - \\
\hline ВC026 & Caciocavallo & ST-111 complex & 800 & + & + & + & + & + & + & + & + & + & + & - \\
\hline ВC009 & Caciocavallo & & 34 & - & - & - & + & + & + & + & + & + & + & - \\
\hline $\mathrm{BC} 0010$ & Caciocavallo & ST-8 complex & 8 & + & + & - & + & + & - & + & + & + & + & - \\
\hline BC0011 & Caciocavallo & ST-142 complex & $2010^{*}$ & + & + & + & + & + & + & + & + & + & + & - \\
\hline $\mathrm{BC} 0012$ & Caciocavallo & & $2002 *$ & + & + & + & + & + & + & - & + & + & + & - \\
\hline ВC0013 & Caciocavallo & & 500 & + & + & + & + & + & + & + & + & - & + & - \\
\hline BC0014 & Caciocavallo & ST-23 complex & $2013^{*}$ & + & + & + & + & + & + & + & + & + & + & - \\
\hline ВC0015 & Caciocavallo & & $2026^{*}$ & + & + & + & + & + & + & - & + & + & - & - \\
\hline $\mathrm{BC} 0016$ & Caciocavallo & & $2028 *$ & - & - & + & + & - & + & - & + & - & - & - \\
\hline ВC0017 & Caciocavallo & ST-365 complex & 1263 & - & - & - & + & + & - & - & + & - & + & - \\
\hline $\mathrm{BC} 0018$ & Caciocavallo & ST-8 complex & 15 & + & + & + & + & + & + & + & + & + & + & - \\
\hline $\mathrm{BC} 0019$ & Caciocavallo & ST-142 complex & 4 & + & + & + & + & + & + & + & + & + & + & + \\
\hline $\mathrm{BC} 0020$ & Caciocavallo & ST-142 complex & 4 & + & + & + & + & + & + & + & + & + & + & - \\
\hline $\mathrm{BC} 0021$ & Caciocavallo & & $2031^{*}$ & + & + & + & + & + & + & + & + & - & + & - \\
\hline BC0022 & Caciocavallo & & 34 & - & - & - & + & + & + & + & + & + & + & - \\
\hline BC0023 & Caciocavallo & ST-23 complex & $2033^{*}$ & + & - & + & + & + & + & - & - & + & + & - \\
\hline $\mathrm{BC} 0025$ & Caciocavallo & & $2036^{*}$ & + & + & + & + & + & + & - & - & + & + & - \\
\hline BC0024 & Cream cheese & & 996 & - & - & - & + & + & - & - & + & + & + & - \\
\hline $\mathrm{BC} 0027$ & Caciocavallo & ST-142 complex & 4 & + & + & + & + & + & + & + & + & + & + & - \\
\hline $\mathrm{BC} 0028$ & Caciocavallo & & 59 & + & - & + & + & + & + & + & + & + & + & - \\
\hline BC0029 & Caciocavallo & & $2041^{*}$ & + & + & + & + & + & + & + & + & + & + & - \\
\hline $\mathrm{BC} 0030$ & Mozzarella & & 369 & - & - & - & + & + & + & + & + & + & + & - \\
\hline BC0031 & Caciocavallo & & $2045^{*}$ & - & - & - & + & + & + & - & - & - & + & + \\
\hline BC0032 & Caciocavallo & & $2045^{*}$ & - & - & - & + & + & + & + & - & + & + & + \\
\hline $\mathrm{BC} 0033$ & Mozzarella & & 12 & + & + & + & + & + & + & + & - & + & + & - \\
\hline BC0034 & Caciocavallo & & $1986^{*}$ & - & + & + & + & + & + & + & + & - & + & + \\
\hline $\mathrm{BC} 0035$ & Mozzarella & & 1420 & + & + & + & + & + & + & + & + & + & + & - \\
\hline $\mathrm{BC} 0036$ & Buffalo mozzarel & & 278 & - & - & + & + & + & + & + & + & + & + & - \\
\hline BC0037 & Caciocavallo & & $1986^{*}$ & + & + & + & + & + & + & + & + & - & + & - \\
\hline BC0038 & Caciocavallo & & $1986 *$ & + & + & + & + & + & + & + & + & - & + & - \\
\hline $\mathrm{BC} 0040$ & Caciocavallo & & $1986^{*}$ & + & + & + & + & + & + & + & - & - & + & - \\
\hline BC0041 & Caciocavallo & ST-23 complex & 197 & + & + & + & + & + & + & + & + & + & + & - \\
\hline BC0042 & Caciocavallo & & $2062 *$ & - & - & + & - & + & + & - & + & - & + & + \\
\hline $\mathrm{BC} 0043$ & Caciocavallo & & $1986^{*}$ & + & + & + & + & + & + & + & + & - & + & - \\
\hline ВC0044 & Caciocavallo & & 262 & + & + & + & + & + & + & + & + & + & + & - \\
\hline BC0045 & Caciocavallo & & $1986^{*}$ & + & + & - & + & + & + & + & + & - & + & - \\
\hline $\mathrm{BC} 0046$ & Caciocavallo & & 2116 & - & - & + & - & - & - & + & - & + & - & - \\
\hline $\mathrm{BC} 0047$ & Caciocavallo & & $1986 *$ & + & + & + & + & + & + & + & + & - & + & - \\
\hline BC0048 & Caciocavallo & ST-205 complex & 205 & + & + & + & + & + & + & + & + & + & + & - \\
\hline BC0049 & Cacioricotta & & 164 & - & - & + & + & + & + & + & + & + & + & - \\
\hline $\mathrm{BC} 0050$ & Mozzarella & & 818 & - & - & + & + & + & + & + & + & + & + & - \\
\hline BC0051 & Mozzarella & & 1097 & - & - & + & + & + & + & - & + & - & + & - \\
\hline $\mathrm{BC} 0052$ & Mozzarella & & 2038 & + & + & + & + & + & + & + & + & + & + & - \\
\hline
\end{tabular}

Continued on the next page. 
$(60 \%)$. Similar results of high prevalence of cytK, entFM and $h b l$ gene complexes in $B$. cereus isolated from milk and dairy products (Reis et al., 2013) and of nhe gene complex, ent $S$ and bceT genes complexes in $B$. cereus isolated from several food and environmental source (i.e. soil) have been described (Yang et al., 2005; Chaves et al., 2012; Yim et al., 2015). When we considered the virulence genes profile and the source analysed, none correlation was found. Whereas when we correlated the ST profiles to virulence genes detected we found nine STs (ST8, ST509, ST996, ST1097, ST1263, ST2028, ST2033,
ST2084 and ST2116) that were associated to an exclusive virulence genes combination. Nevertheless, this genetic profile correlation is questionable, considering that we identified one strain for each of nine STs previously mentioned. Thus, further studies will be necessary for demonstrated really correlation between MLST and virulence genes profiles.

Based on the results and considering the genetic diversity of $B$. cereus s.l., the MLST approach has proven to be an accurate method for strain genetic typing. Interestingly, our analysis demonstrated the presence of toxin genes in all strains of the
B. cereus s.l., stressing the their potentially pathogenic power. Taken together, these data appear to be of significant interest for public health, since highlight potential risk connected to ready-to-eat foods, generally consumed without cooking. In order to prevent contamination by B. cereus s.l., it is very important observe the correct hygiene practices along food chain. In addition, it would be advisable to implement scrupulous control measures of food preservation, to prevent the multiplication of the microorganism and the consequent production of toxins.

Table 2. Continued from previous page.

\begin{tabular}{|c|c|c|c|c|c|c|c|c|c|c|c|c|c|c|}
\hline \multirow{2}{*}{\multicolumn{2}{|c|}{ ID sample Source }} & \multirow[t]{2}{*}{$\mathrm{CC}$} & \multirow[t]{2}{*}{ ST } & \multicolumn{11}{|c|}{ Virulence genes } \\
\hline & & & & hblA & hblc & hblD & nheA & nheB & nheC & cytK & entFM & bceT & entS & ces \\
\hline $\mathrm{BC} 0053$ & Caciocavallo & & $1986^{*}$ & + & + & + & + & + & + & + & + & - & + & - \\
\hline $\mathrm{BC} 0054$ & Caciocavallo & & 26 & + & + & + & + & + & + & + & + & + & + & - \\
\hline $\mathrm{BC} 0055$ & Caciocavallo & & $1986^{*}$ & + & + & + & + & + & + & + & + & - & + & - \\
\hline $\mathrm{BC} 0056$ & Butter & & 120 & + & - & + & + & + & + & + & + & + & + & - \\
\hline $\mathrm{BC} 0057$ & Mozzarella & & 1665 & + & - & + & + & + & + & + & + & - & + & - \\
\hline $\mathrm{BC} 0058$ & Caciocavallo & & $1986^{*}$ & + & + & + & + & + & + & + & + & - & + & - \\
\hline BC0059 & Caciocavallo & ST-142 complex & 4 & + & + & + & + & + & + & + & + & - & + & - \\
\hline $\mathrm{BC} 0060$ & Mozzarella & ST-97 complex & 1578 & + & + & + & + & + & + & + & + & - & + & - \\
\hline BC0061 & Caciocavallo & & $1986 *$ & + & + & + & + & + & + & + & + & - & + & - \\
\hline $\mathrm{BC} 0062$ & Caciocavallo & & 500 & + & + & + & + & + & + & + & + & - & + & - \\
\hline $\mathrm{BC} 0063$ & Mozzarella & & 1097 & - & + & - & + & + & + & - & + & - & + & - \\
\hline $\mathrm{BC} 0064$ & Sheep's cheese & & 1063 & - & - & + & + & + & + & - & + & + & + & - \\
\hline BC0065 & Caciotta & & 12 & - & - & + & + & + & + & - & + & + & + & - \\
\hline BC0066 & Mozzarella & & 12 & - & - & + & + & - & - & + & - & - & + & - \\
\hline $\mathrm{BC} 0067$ & Caciocavallo & & 371 & + & + & + & + & + & + & + & + & + & + & - \\
\hline BC0068 & Mozzarella & & 1007 & - & - & - & + & + & + & + & + & + & + & - \\
\hline BC0069 & Caciocavallo & & 92 & + & + & + & + & + & + & + & + & + & + & - \\
\hline BC0070 & Caciocavallo & & $1986^{*}$ & + & + & + & + & + & + & + & + & - & + & - \\
\hline BC0071 & Mozzarella & & 92 & + & + & + & + & + & + & + & - & + & + & - \\
\hline BC0072 & Mozzarella & & 2261 & + & + & + & + & + & + & + & + & + & + & - \\
\hline ВC008 & Caciocavallo & & 632 & + & + & + & + & + & + & - & + & + & - & - \\
\hline BC0073 & Mozzarella & ST-111 complex & 562 & - & - & - & + & + & + & - & + & - & + & - \\
\hline $\mathrm{BC} 0074$ & Mozzarella & & 92 & + & + & + & - & + & - & + & + & + & - & - \\
\hline $\mathrm{BC} 0075$ & Sheep's cheese & & 1223 & - & - & - & + & + & + & - & + & - & - & - \\
\hline ВC0076 & Mozzarella & & 24 & - & - & + & + & + & + & - & - & + & + & - \\
\hline BC0077 & Mozzarella & & 92 & + & + & + & - & + & + & + & - & + & + & - \\
\hline $\mathrm{BC} 0078$ & Caciocavallo & & $2083^{*}$ & + & + & + & + & + & + & + & + & + & + & - \\
\hline BC0079 & Caciocavallo & ST-365 complex & $2084^{*}$ & - & + & + & + & - & + & - & + & - & + & - \\
\hline $\mathrm{BC} 0080$ & Milk & & 56 & + & + & + & + & + & + & + & + & + & + & - \\
\hline BC0081 & Caciocavallo & & $1833^{*}$ & + & + & + & + & + & + & - & + & + & + & - \\
\hline BC0082 & Butter & & 1355 & + & + & + & + & + & + & + & + & + & + & - \\
\hline $\mathrm{BC} 0083$ & Caciocavallo & & $1967^{*}$ & + & + & + & + & + & + & + & + & + & + & - \\
\hline ВC0084 & Mozzarella & & 414 & - & - & - & + & + & + & + & + & + & + & - \\
\hline BC0085 & Mozzarella & & 142 & + & + & + & + & + & + & + & + & + & + & - \\
\hline $\mathrm{BC} 0086$ & Mozzarella & & 24 & + & + & + & + & + & + & + & + & + & + & - \\
\hline BC0087 & Caciocavallo & & $1967^{*}$ & + & + & + & + & + & + & + & + & + & + & - \\
\hline BC0088 & Milk & & 1810 & + & + & + & + & + & + & - & + & + & + & - \\
\hline ВC0089 & Yogurt & & 142 & + & - & - & - & + & + & - & + & + & + & - \\
\hline BC0090 & Caciocavallo & & $2095^{*}$ & + & + & + & + & + & + & + & + & + & + & - \\
\hline BC0094 & Caciocavallo & & $2036^{*}$ & + & + & + & - & - & - & - & + & + & - & - \\
\hline
\end{tabular}




\section{References}

Agata N, Ohta M, Arakawa Y, Mori M, 1995. The bceT gene of Bacillus cereus encodes an enterotoxic protein. Microbiol 141:983-8.

Agata N, Ohta M, Yokoyama K, 2002. Production of Bacillus cereus emetic toxin (cereulide) in various foods. Int J Food Microbiol 73:23-7.

Asano SI, Nukumizu Y, Bando H, Iizuka T, Yamamoto T, 1997. Cloning of novel enterotoxin genes from Bacillus cereus and Bacillus thuringiensis. Appl. Environ. Microbiol 63:1054-7.

Beecher DJ, Wong AC, 1994. Identification and analysis of the antigens detected by two commercial Bacillus cereus diarrheal enterotoxin immunoassay kits. Appl. Environ. Microbiol 60:4614-6.

Beuchat LR, Ma-Lin CF, Carpenter JA, 1980. Growth of Bacillus cereus in media containing plant seed materials and ingredients used in Chinese cookery. J Appl Bacteriol 48:397-407.

Brillard J, Dupont CMS, Berge O, Dargaignaratz $\mathrm{C}$, Oriol-Gagnier $\mathrm{S}$, Doussan C, Broussolle V, Gillon M, Clavel T, Bérard A, 2015. The water cycle, a potential source of the bacterial pathogen Bacillus cereus. Biomed Res Int 2015:356928.

Cardazzo B, Negrisolo E, Carraro L, Alberghini L, Patarnello T, Giaccone V, 2008. Multiple-locus sequence typing and analysis of toxin genes in Bacillus cereus food-borne isolates. Appl Environ Microbiol 74:850-60.

Castiaux V, Laloux L, Schneider YJ, Mahillon J, 2016. Screening of Cytotoxic B. cereus on Differentiated Caco-2 Cells and in Co-Culture with Mucus-Secreting (HT29-MTX) Cells. Toxins 5:8(11).

Chang YH, Shangkuan YH, Lin HC, Liu HW, 2003. PCR assay of the groEL gene for detection and differentiation of Bacillus cereus group cells. Appl Environ Microbiol 69:4502-10.

Chaves, JQ, Cavados C de FG, Vivoni AM, 2012. Molecular and toxigenic characterization of Bacillus cereus and Bacillus thuringiensis strains isolated from commercial ground roasted coffee. J Food Prot 75:518-22.

Christiansson A, Bertilsson J, Svensson B, 1999. Bacillus cereus spores in raw milk: factors affecting the contamination of milk during the grazing period. $\mathrm{J}$ Dairy Sci 82:305-14.
Didelot X, Barker M, Falush D, Priest FG, 2009. Evolution of pathogenicity in the Bacillus cereus group. Syst Appl Microbiol 32:81-90.

Ehling-Schulz M, Svensson B, Guinebretiere $\mathrm{MH}$, Lindbäck T, Andersson M, Schulz A, Fricker M, Christiansson A, Granum PE, Märtlbauer E, Nguyen-The C, Salkinoja-Salonen M, Scherer S, 2005. Emetic toxin formation of Bacillus cereus is restricted to a single evolutionary lineage of closely related strains. Microbiol 151:183-97.

Fagerlund A, Ween O, Lund T, Hardy SP, Granum PE, 2004. Genetic and functional analysis of the cytK family of genes in Bacillus cereus. Microbiol 150:2689-97.

Fricker M, Messelhäusser U, Busch U, Scherer S, Ehling-Schulz M, 2007. Diagnostic real-time PCR assays for the detection of emetic Bacillus cereus strains in foods and recent food-borne outbreaks. Appl Environ Microbiol 73:1892-8.

Ghelardi E, Celandroni F, Salvetti S, Barsotti C, Baggiani A, Senesi S, 2002. Identification and characterization of toxigenic Bacillus cereus isolates responsible for two food-poisoning outbreaks. FEMS Microbiol Lett 208:12934.

Granum PE, Lund T, 1997. Bacillus cereus and its food poisoning toxins. FEMS Microbiol Lett 157:223-8.

Guinebretière MH, Broussolle V, NguyenThe C, 2002. Enterotoxigenic profiles of food-poisoning and food-borne Bacillus cereus strains. J Clin Microbiol 40:3053-6.

Harmon SM, Kautter DA, 1991. Incidence and growth potential of Bacillus cereus in ready-to-serve foods. J Food Prot 54:372-4.

Hendriksen NB, Hansen BM, Johansen JE, 2006. Occurrence and pathogenic potential of Bacillus cereus group bacteria in a sandy loam. Antonie Van Leeuwenhoek 89:239-49.

López AC, Minnaard J, Pérez PF, Alippi AM, 2015. A case of intoxication due to a highly cytotoxic Bacillus cereus strain isolated from cooked chicken. Food Microbiol 46:195-9.

Madeira JP, Alpha-Bazin B, Armengaud J, Duport C, 2015. Time dynamics of the Bacillus cereus exoproteome are shaped by cellular oxidation. Front Microbiol $6: 342$.

Owusu-Kwarteng J, Wuni A, Akabanda F,
Tano-Debrah K, Jespersen L, 2017.

Prevalence, virulence factor genes and antibiotic resistance of Bacillus cereus sensu lato isolated from dairy farms and traditional dairy products. BMC Microbiol 17:65.

Priest FG, Barker M, Baillie LWJ, Holmes EC, Maiden MCJ, 2004. Population structure and evolution of the Bacillus cereus group. J Bacteriol 186:7959-70.

Reis ALS, Montanhini MTM, Bittencourt JVM, Destro MT, Bersot LS, 2013. Gene detection and toxin production evaluation of hemolysin BL of Bacillus cereus isolated from milk and dairy products marketed in Brazil. Braz. J Microbiol 44:1195-8.

Sergeev N, Distler M, Vargas M, Chizhikov V, Herold KE, Rasooly A, 2006. Microarray analysis of Bacillus cereus group virulence factors. J Microbiol Methods 65:488-502.

Wetterhall M, Grönberg A, Grönlund S, Björkman T, Sandberg L, Musunuri S, Chaloupka K, Gammell P, 2019. Removal of B. cereus cereulide toxin from monoclonal antibody bioprocess feed via two-step Protein A affinity and multimodal chromatography. J Chromatogr B Analyt Technol Biomed Life Sci 1118-1119:194-202.

Yang IC, Shih DYC, Huang TP, Huang YP, Wang JY, Pan TM, 2005. Establishment of a novel multiplex PCR assay and detection of toxigenic strains of the species in the Bacillus cereus group. $\mathrm{J}$ Food Prot 68:2123-30.

Yang Y, Yu X, Zhan L, Chen J, Zhang Yunyi, Zhang J, Chen H, Zhang Z, Zhang Yanjun, Lu Y, Mei L, 2017. Multilocus sequence type profiles of Bacillus cereus isolates from infant formula in China. Food Microbiol 62:4650.

Yim JH, Kim K Y, Chon JW, Kim DH, Kim HS, Choi DS, Choi IS, Seo KH, 2015. Incidence, antibiotic susceptibility, and toxin profiles of Bacillus cereus sensu lato Isolated from Korean fermented soybean products. J Food Sci 80:M1266-70. 